\title{
Secondary infertility in women: radiologic evaluation
}

\author{
This article was published in the following Dove Press journal: \\ Reports in Medical Imaging \\ 10 January $201 \mathrm{I}$ \\ Number of times this article has been viewed
}

\author{
Jeffrey Dee Olpin \\ Anne Kennedy \\ University of Utah Health Sciences \\ Center, Salt Lake City, UT, USA
}

Correspondence: Jeffrey Olpin University of Utah Health Sciences Center, 30 N. 1900 e. \#I A7I, Salt Lake City, UT 84I 32, USA

$\mathrm{Tel}+\mathrm{I} 80158 \mid 7553$

Fax + I 80I 58I 24I4

Email jeffrey.olpin@hsc.utah.edu

\begin{abstract}
Female infertility is a commonly encountered problem that presently accounts for a significant percentage of women seeking gynecologic services. While primary infertility is defined as the inability to conceive or carry a pregnancy successfully to full term, secondary infertility is defined as difficulty in conceiving after already having previously conceived (either carrying a pregnancy to term or a miscarriage). The causes of both primary and secondary female infertility are varied, and include various disorders involving the fallopian tubes, ovaries, uterus, cervix, and peritoneum. Imaging has become an essential tool in the workup of female infertility. Various imaging modalities are commonly employed to evaluate the female reproductive tract. Hysterosalpingography is typically performed as a baseline imaging study in the workup of female infertility. Ultrasound and pelvic magnetic resonance imaging studies are likewise routinely utilized to aid in the diagnosis of female infertility. The appropriate selection of imaging modalities is essential in establishing the etiology of female infertility in a timely, efficient, and cost-effective manner.
\end{abstract}

Keywords: female secondary infertility, reproductive system, imaging, radiologic evaluation

\section{Introduction}

Female infertility is a commonly encountered problem that can be both financially and emotionally challenging for many couples. There are an estimated 7.4 million women, or $12 \%$ of the female population of reproductive age in the US who are reportedly classified as infertile, according to the 2002 National Survey of Family Growth. ${ }^{1}$ There has been a steadily increasing demand for infertility services between 1996 and 2004, with a $92 \%$ increase in the number of assisted reproduction procedures. ${ }^{2}$ In general, male medical conditions (sperm disorders) account for roughly $35 \%$ of infertility cases. Decreased ovarian reserve or primary ovulatory dysfunction accounts for roughly $20 \%$ of infertility cases. Abnormal cervical mucus or unidentified factors account for nearly $15 \%$ of infertility cases. The remaining $30 \%$ of cases are due to primary structural disorders of the female reproductive system, for which imaging plays a crucial role.

Secondary infertility is defined as difficulty in conceiving after already having previously conceived (either carrying a pregnancy to term or a miscarriage). Along with the recent rise in demand for infertility services, there has been an increased demand for female infertility imaging, which has become an indispensible tool in the workup of secondary female infertility. There are multiple disorders of the female reproductive tract that can contribute to either primary or secondary infertility.

Various imaging modalities are commonly employed in the workup of female infertility, including hysterosalpingography, ultrasound, computed tomography (CT), submit your manuscript | www.dovepress.com

Dovepress

DOI: 10.2147/RMI.S9179 
and magnetic resonance imaging. Hysterosalpingography provides the most effective modality in the evaluation of the fallopian tubes. Although hysterosalpingography can provide valuable information regarding the endometrium, ultrasound is generally employed as the first-line imaging modality in the evaluation of the endometrium. Newer three-dimensional sonographic techniques have recently been developed that can provide valuable detail of complex uterine pathology. Magnetic resonance has revolutionized pelvic imaging in recent decades, and is considered the gold standard for evaluation of many conditions of the female reproductive tract.

A fertility workup commonly begins with a thorough history and physical of both partners. Standard ovulation and ovarian reserve testing is routinely performed as part of a female infertility workup. Hysterosalpingography is commonly performed as part of a routine infertility evaluation. Hysterosalpingography serves as a baseline imaging study for the detection of a variety of gynecologic disorders. Hysterosalpingography may assist in not only detecting disorders of the fallopian tubes, but may also suggest other disorders of the female reproductive system. Additional imaging modalities can then be employed for further evaluation in a systematic fashion, depending on the hysterosalpingography findings. This article discusses the use of multimodal imaging for diagnosing disorders of the fallopian tube, ovary, uterus, cervix, and peritoneum that contribute to secondary female infertility.

\section{Disorders of the fallopian tube}

Fallopian tube disorders account for up to $30 \%-40 \%$ of cases of structural female infertility. Abnormalities of the fallopian tube are considered the most common cause of female infertility. ${ }^{3}$ The fallopian tube is roughly $10-12 \mathrm{~cm}$ in length, and serves as a conduit for the ovum to travel from the ovary to the endometrial cavity. The fallopian tube is anatomically subdivided into four segments from lateral to medial, ie, the fimbriated infundibular portion nearest the ovary, the ampullary region which represents the longest portion of the lateral tube, the isthmus which represents the narrower part of the tube near the uterus, and the interstitial or intramural portion of the tube that traverses the uterine myometrium. Occlusion can occur at any location along the length of the tube.

As previously stated, hysterosalpingography is the primary imaging modality for the evaluation of the fallopian tubes, whereas laparoscopy provides the most accurate means of assessing the peritubal region. ${ }^{3}$ Other fallopian tube imaging techniques have been described, including contrast-enhanced sonohysterography. However, this technique requires the use of ultrasound contrast agents, which are currently not approved by the US Food and Drug Administration. ${ }^{4}$ CT hysterosalpingography has recently been described for the evaluation of both uterine and tubal disorders. ${ }^{5}$ Unlike other imaging modalities, this technique has the advantage of being able to depict both the external and internal uterine contour, as well as the fallopian tubes and other pelvic structures in one comprehensive examination. A disadvantage is the use of ionizing radiation. Magnetic resonance hysterography has likewise been suggested as a means of assessing tubal patency as a result of a study using heavily $\mathrm{T}_{2}$-weighted sequences through the pelvis similar to that of magnetic resonance cholangiopancreatography. ${ }^{6}$ However, most normal fallopian tubes are too narrow in caliber to be visualized by routine magnetic resonance.

A hysterosalpingography is performed by placing a sterile catheter through the cervical os into the endometrial cavity. Radio-opaque contrast material is then injected into the endometrial cavity under direct fluoroscopic observation. Contrast medium is typically hand injected, with the goal of achieving free spillage of contrast material from the fallopian tubes (Figure 1). Hysterosalpingography contraindications include active pelvic inflammatory disease and pregnancy. At hysterosalpingography, the fallopian tube is a very thin, curvilinear structure that gradually widens in the ampullary portion. Fallopian tubes vary in location within the pelvis and in the degree of inherent tortuosity. ${ }^{7}$ The cornual portion of the fallopian tube is surrounded by smooth muscle of the uterus. Tubal spasm is commonly encountered at hysterosalpingography, and is manifested by tubal occlusion within this region of the tube. Spasm is a common cause of false

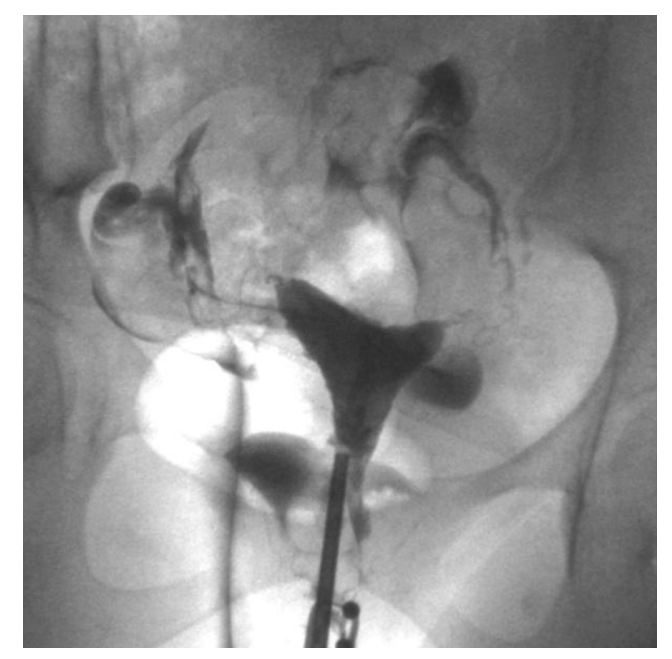

Figure I Normal hysterosalpingography. Hysterosalpingography from an initial infertility workup in a 32-year-old female demonstrates a normal contour endometrial cavity. Free spillage of contrast material is seen into the peritoneal cavity from both fallopian tubes. 


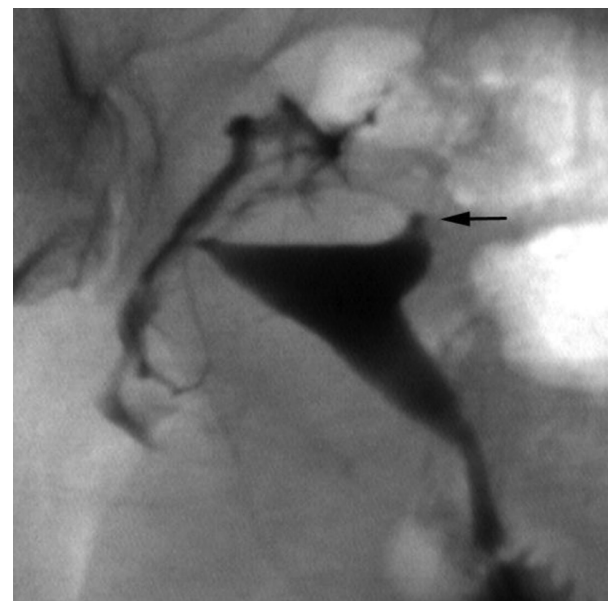

Figure 2 Left tubal occlusion. Hysterosalpingography performed for routine infertility workup in a 28-year-old female demonstrates abrupt occlusion of the proximal left fallopian tube in this patient with a recently documented history of pelvic inflammatory disease.

positive tubal obstruction, and must be differentiated from genuine tubal obstruction. Some have advocated the use of a spasmolytic agent, such as glucagon, in order to prevent tubal spasm. Others advocate that turning the patient prone may aid in relieving tubal spasm. ${ }^{7}$ Delayed injection of contrast material is often necessary after spasm has resolved. ${ }^{8}$

Pelvic inflammatory disease is a leading cause of tubal occlusion and is frequently seen in the setting of secondary infertility. Pelvic inflammatory disease afflicts over one million women and accounts for over 275,000 hospitalizations each year. ${ }^{9}$ Primary clinical symptoms include fever and pelvic pain. Less common symptoms include vaginal discharge, uterine bleeding, dyspareunia, dysuria, as well as adnexal or cervical tenderness. ${ }^{10}$ The most common pathogens include Neisseria gonorrhoeae and Chlamydia trachomatis, although $30 \%-40 \%$ of cases are polymicrobial. ${ }^{10}$ Risk factors for pelvic inflammatory disease include young age, sexual promiscuity, low socioeconomic status, frequent douching, the presence of an intrauterine device, as well as other pelvic instrumentation. ${ }^{11}$ Untreated pelvic inflammatory disease can lead to tubal scarring and eventual occlusion. Patients with chronic pelvic inflammatory disease have an approximately six-fold higher risk for ectopic pregnancy and chronic pelvic pain. ${ }^{9}$

The gold standard for the diagnosis of pelvic inflammatory disease is laparoscopy, which provides direct visualization of inflamed pelvic structures. ${ }^{11}$ However, laparoscopy is invasive and expensive, and is less frequently used in recent decades given the ready availability of ultrasound and CT. Early radiographic manifestations of salpingitis include generalized thickening of the fallopian tubes and tubal obstruction (Figure 2). Other manifestations of early pelvic inflammatory

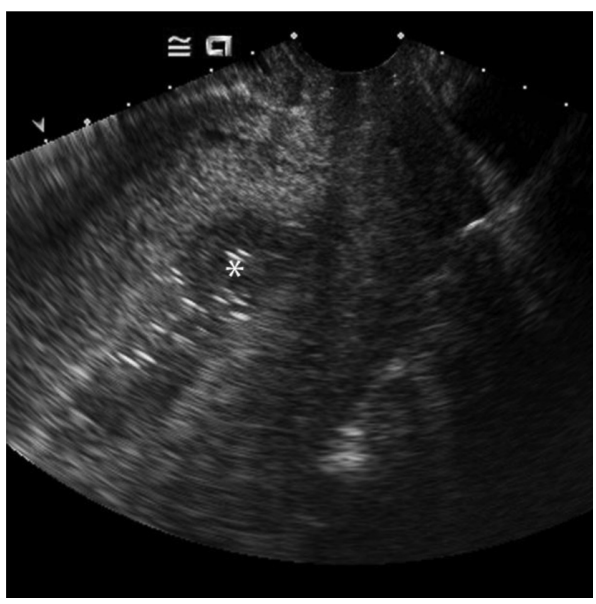

Figure 3 Endometritis. Longitudinal image from a transvaginal sonogram in a 35-yearold female with pelvic inflammatory disease demonstrates thickened, heterogeneous endometrium $(*)$. Fluid and internal echoes indicative of intraluminal gas are likewise noted.

disease include pelvic edema with generalized haziness of the pelvic fat and obscuration of fascial planes. Mild oophoritis can likewise be seen with heterogeneously enlarged ovaries. Endometritis can be seen in the setting of pelvic inflammatory disease, and can often be diagnosed on ultrasound by the presence of gas and fluid within the endometrial cavity (Figure 3). Endometritis is readily diagnosed on CT by the presence of endometrial fluid and gas, as well as abnormal endometrial enhancement (Figure 4). Radiographic manifestations of more advanced or chronic pelvic inflammatory disease include high-grade tubal occlusion. A pyosalpinx is a commonly encountered radiographic manifestation of tubal occlusion, which involves dilatation of the fallopian tube, usually along its entire course as a result of distal tubal

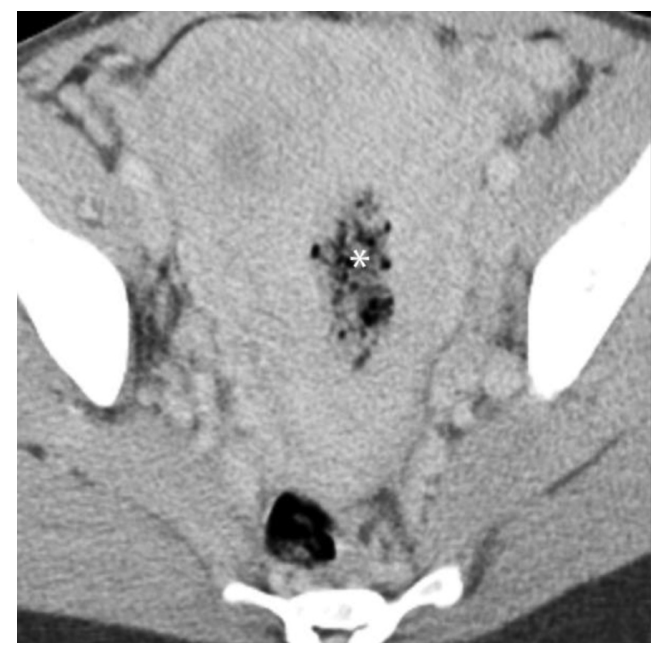

Figure 4 Endometritis. Noncontrast computed tomography scan from the same patient in Figure 3 confirms the presence of gas within the endometrium (*). 


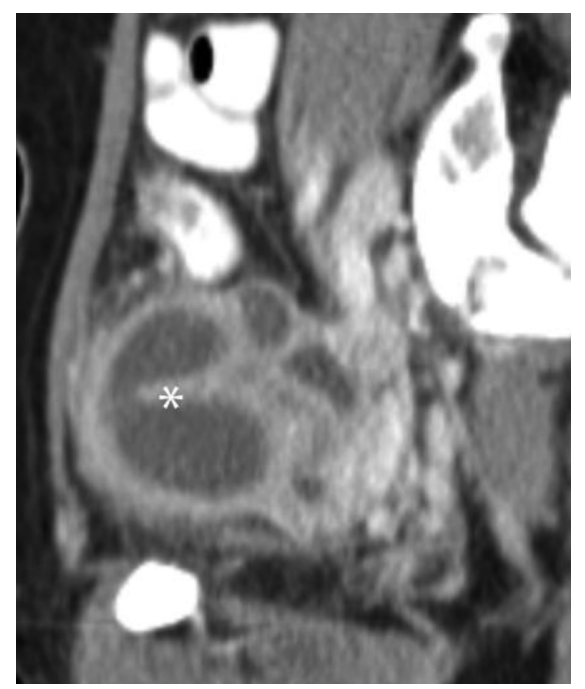

Figure 5 Pyosalpinx. Sagittal contrast enhanced computed tomography image from a 30-year-old female with pelvic inflammatory disease. A tubular structure is noted within the left pelvis with thickened, enhancing walls and indistinct margins $\left(^{*}\right)$. These findings are consistent with acute pyosalpinx.

obstruction (Figure 5). The occlusion may be unilateral or bilateral, and may affect any portion of the fallopian tube (Figures 6 and 7).

Tubo-ovarian abscesses are often seen as loculated peritubal fluid collections adjacent to the distal or ampullary portions of the fallopian tubes (Figures 8 and 9). Tubo-ovarian abscesses are often thick-walled with ill-defined, irregular margins (Figure 10). Tubo-ovarian abscesses frequently result in peritubal adhesions, which are an important cause of secondary infertility. ${ }^{12}$ Adjacent inflammatory changes of

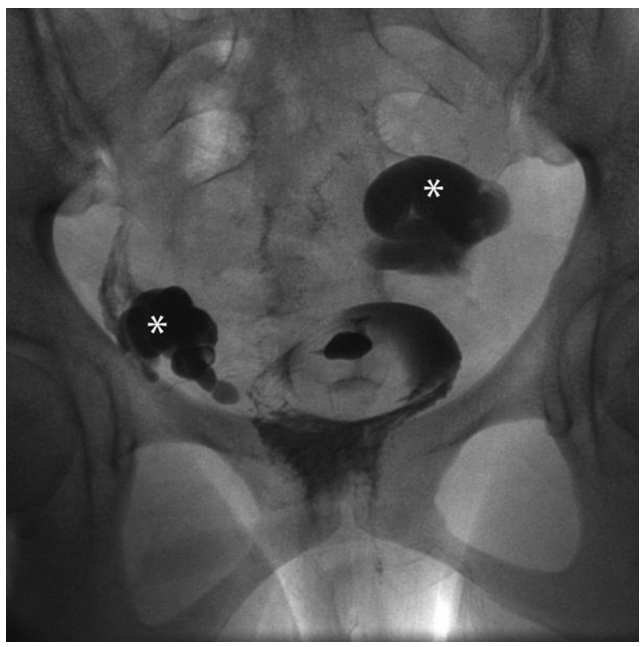

Figure 6 Bilateral hydrosalpinges. Hysterosalpingography from a 32-year-old women with a history of prior pelvic inflammatory disease. The fallopian tubes are dilated along their entire course bilaterally $(*)$. No spillage of contrast was observed from either tube.

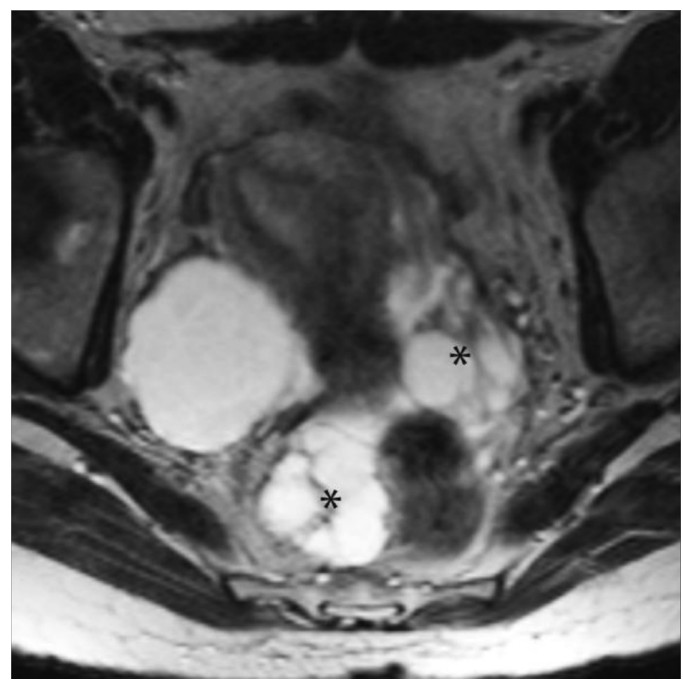

Figure 7 Bilateral hydrosalpinges. Axial $\mathrm{T}_{2}$-weighted magnetic resonance image from the same patient in Figure 6 demonstrates dilated, fluid-filled fallopians tubes $(*)$.

the small bowel and colon may occasionally be seen. More remote inflammatory changes from pelvic inflammatory disease, otherwise known as Fitz-Hugh-Curtis syndrome, consist of inflammatory changes within the right upper quadrant. In this syndrome, bacteria spread to the right upper quadrant via the right paracolic gutter or via the lymphatics. CT manifestations of this condition include heterogeneous enhancement of the liver capsule and generalized inflammatory changes in the gallbladder fossa (Figure 11). ${ }^{11,13}$

A relatively common cause of tubal occlusion leading to secondary infertility includes tubal scarring from prior

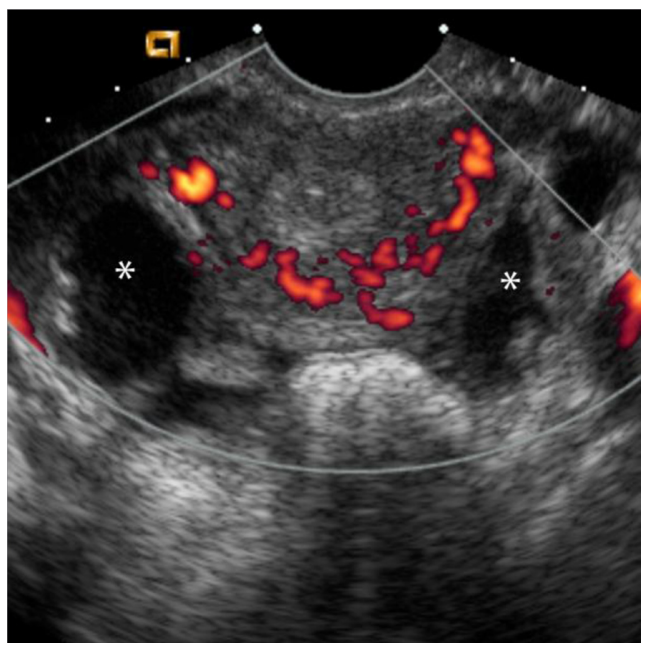

Figure 8 Tubo-ovarian abscesses. Transverse image from a transvaginal sonogram in a 24-year-old woman with known pelvic inflammatory disease. Dilated fallopian tubes are noted bilaterally $(*)$ with associated complex adnexal masses. 


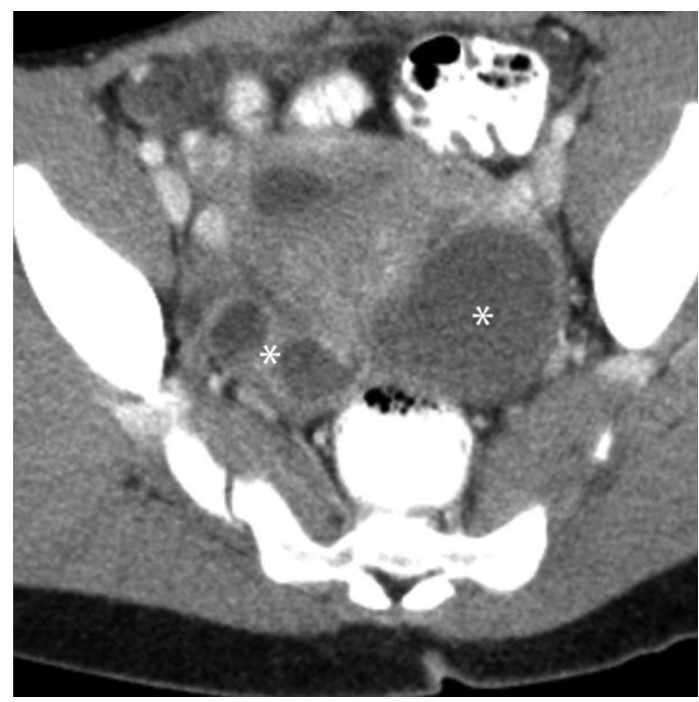

Figure 9 Tubo-ovarian abscesses. Contrast-enhanced computed tomography scan from the same patient in Figure 8 demonstrates dilated fallopian tubes with associated complex adnexal masses $(*)$.

pelvic surgery. Less common etiologies include intratubal endometriosis, fallopian tube carcinoma, and granulomatous salpingitis from tuberculous infection (Figure 12). Congenital tubal atresia generally results in primary female infertility, but is rarely if ever seen in the setting of secondary female infertility. Treatment options for tubal occlusion include transcervical fallopian tube recanalization under fluoroscopic guidance. Tubal microsurgery may also be an effective option in the event that transcervical recanalization is unsuccessful.

Nonocclusive tubal disorders can be seen in the setting of salpingitis isthmica nodosa, an inflammatory process of the

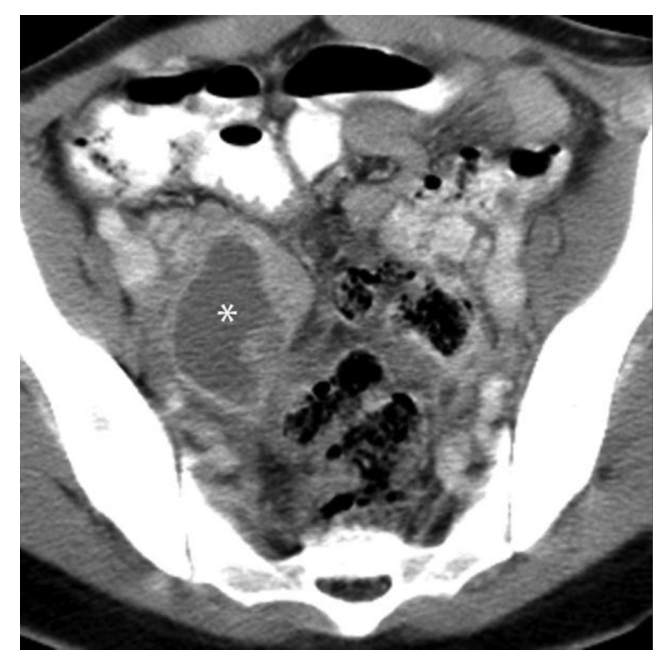

Figure 10 Tubo-ovarian abscess. Contrast-enhanced computed tomography image from a 31-year-old female with pelvic inflammatory disease. A cystic mass is noted arising from the right adnexa with thickened, irregular walls $(*)$.

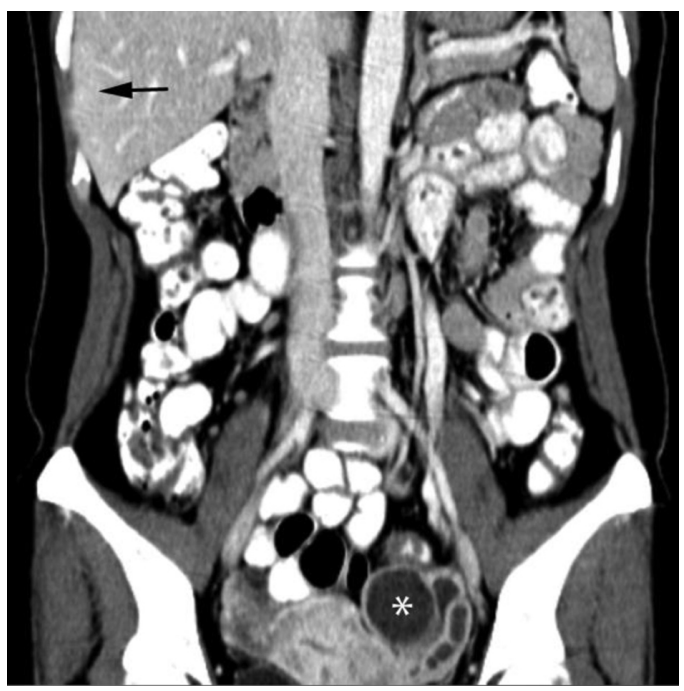

Figure I I Fitz-Hugh-Curtis syndrome. Coronal contrast-enhanced computed tomography image from a 27-year-old female with fever and right upper quadrant abdominal pain. An ill-defined area of capsular enhancement and capsular irregularity is noted along the lateral aspect of the right lobe of the liver (arrow). A pyosalpinx is likewise noted arising from the left adnexa $(*)$.

fallopian tube. The etiology is unclear, although the disorder appears to be associated with pelvic inflammatory disease, and rarely, ectopic pregnancy. Secondary infertility is often seen as a result of this condition. Hysterosalpingography findings of salpingitis isthmica nodosa include tubal irregularity with associated diverticular outpouchings from the tube that typically involves the medial or isthmic portion of the fallopian tube (Figure 13). Salpingitis isthmica nodosa may involve one or both fallopian tubes. Patients with salpingitis isthmica nodosa are generally not candidates for fallopian tube

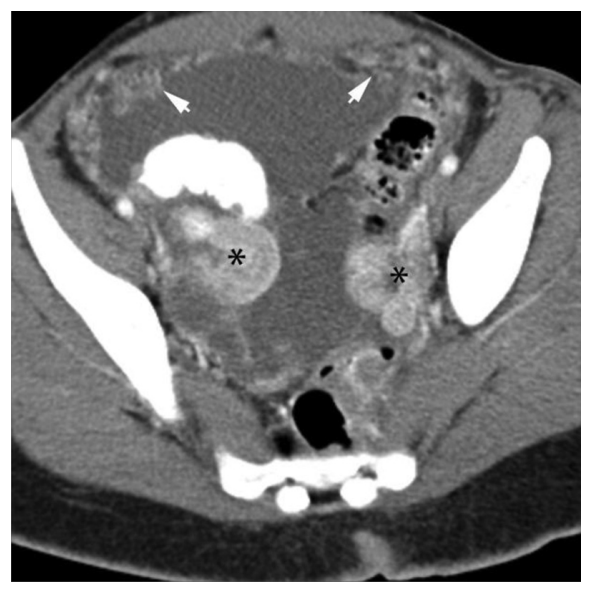

Figure 12 Granulomatous salpingitis from tuberculous peritonitis. Contrastenhanced computed tomography image from a 33-year-old female with systemic tuberculosis. Thickened, heterogeneously enhancing fallopian tubes are noted bilaterally $(*)$. Diffuse stippling and nodularity of the omentum is likewise noted (arrows) with associated pelvic ascites, consistent with tuberculous peritonitis. 


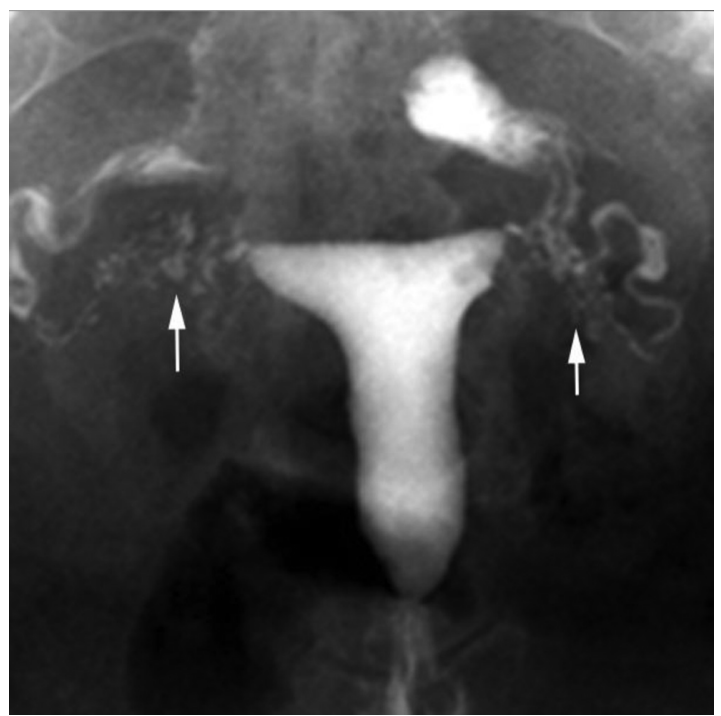

Figure 13 Salpingitis isthmica nodosa. Hysterosalpingography from a 29-year-old female with a history of chronic pelvic inflammatory disease. Multiple irregular diverticular outpouchings (arrows) are seen arising the proximal aspect of both fallopian tubes. Bilateral tubal occlusion was likewise noted.

recanalization, and are typically offered in vitro fertilization as an alternative method of assisted reproduction.

\section{Disorders of the ovary}

Primary ovarian abnormalities associated with infertility include nonfunctional ovaries, and premature ovarian failure. These disorders are typically diagnosed by clinical and biochemical parameters. However, other conditions of the ovary, such as gonadal dysgenesis, polycystic ovarian syndrome, and ovarian neoplasms are readily diagnosed by imaging. Pelvic ultrasound is generally considered the initial imaging modality of choice for the evaluation of the ovaries.

Gonadal dysgenesis is a congenital developmental disorder of the female reproductive system that results in extremely hypoplastic or absent ovaries. The absence of ovaries is readily apparent on imaging studies, although this condition plays little role in the workup of secondary infertility.

Polycystic ovarian syndrome is a leading cause of infertility, affecting up to $8 \%$ of women. ${ }^{14}$ However, not all women with polycystic ovarian syndrome have difficulty in becoming pregnant. The condition accounts for a much higher percentage of primary infertility as opposed to secondary infertility. Clinical manifestations of polycystic ovarian syndrome include obesity, hirsutism, and oligomenorrhea. Polycystic ovarian syndrome is the result of hyperandrogenism, which leads to elevated serum levels of luteinizing hormone and morphologic changes of the ovary, which can be seen in up to $80 \%$ of women with this disorder. ${ }^{14}$ Ultrasound features

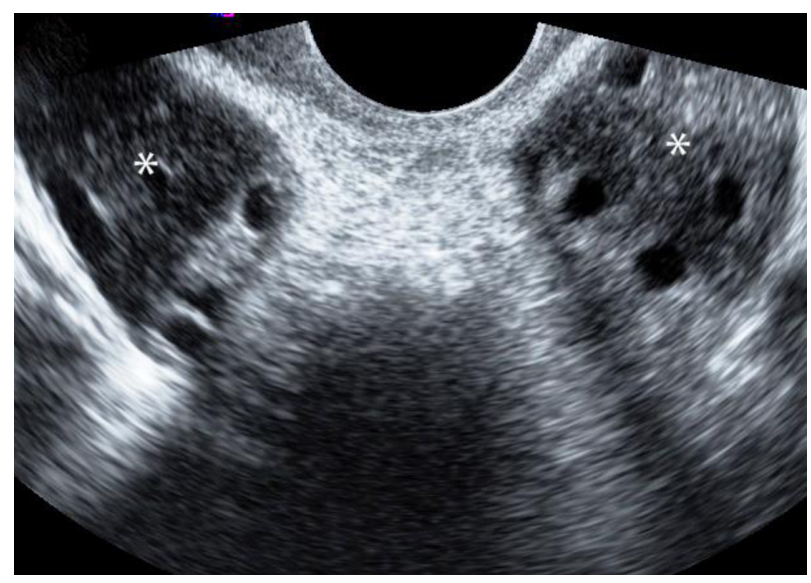

Figure I4 Polycystic ovarian syndrome. Transverse image from a transvaginal pelvic sonogram in a 32-year-old female with oligomenorrhea and infertility. There is bilateral ovarian enlargement (*). Multiple small, peripheral follicles are noted with a relative paucity of central follicles.

of polycystic ovarian syndrome include enlarged ovaries, increased number of uniformly small peripheral follicles, and increased echogenicity of the ovarian stroma (Figure 14). Similar features can likewise be seen on CT, although this imaging modality is not routinely used for the evaluation of polycystic ovarian syndrome (Figure 15). Magnetic resonance features include reduced signal-intensity central stroma with hyperintense peripheral follicles on $\mathrm{T}_{2}$-weighted images (Figure 16).

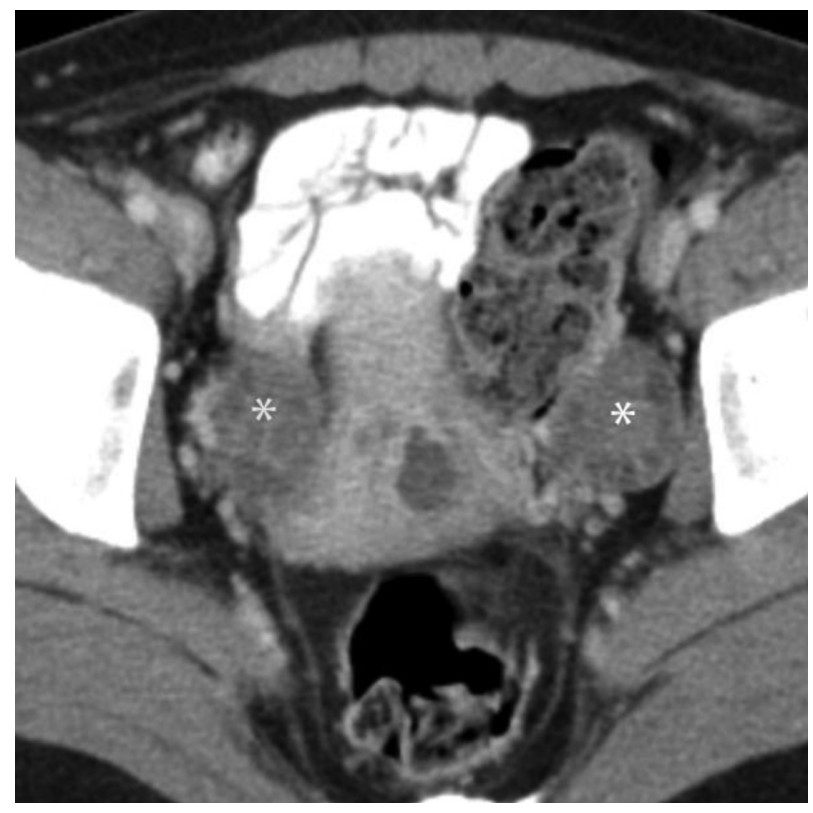

Figure I 5 Polycystic ovarian syndrome. Contrast-enhanced computed tomography scan from the same patient in Figure 14 demonstrates uniformly enlarged ovaries bilaterally. 


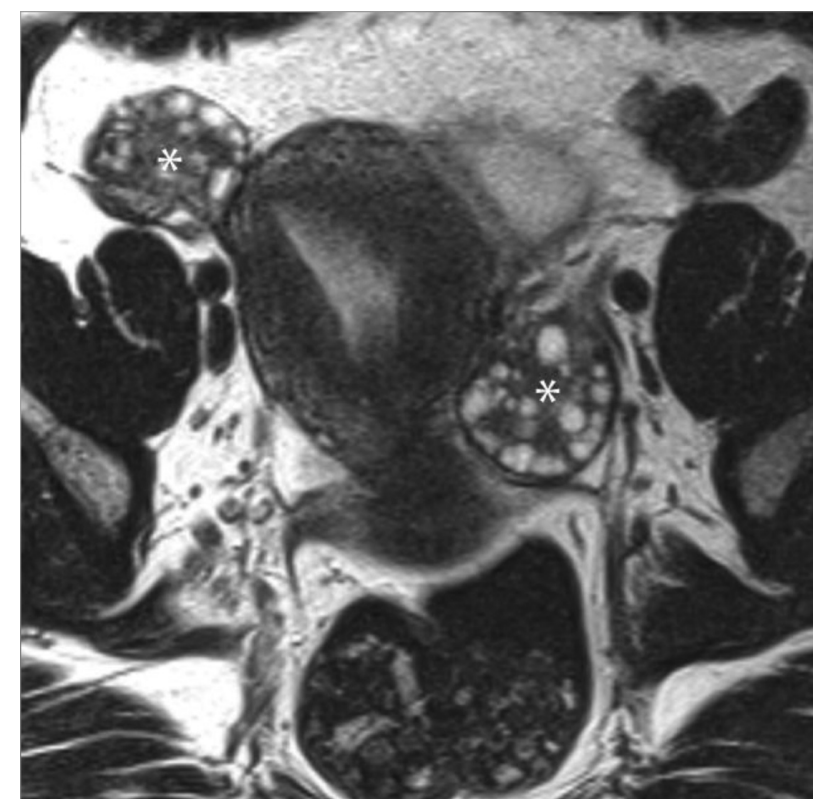

Figure 16 Polycystic ovarian syndrome. Axial $\mathrm{T}_{2}$-weighted magnetic resonance image in a 34-year-old female with hirsutism and amenorrhea. Bilateral ovarian enlargement is noted $(*)$ with multiple small, peripheral follicles.

Ovarian masses encompass a broad range of benign and malignant lesions. While ovarian masses are commonly encountered in a clinical gynecologic setting, they are rarely implicated in the setting of secondary infertility. Ovarian masses have been known to result anecdotally in reduced fertility due to mass effect from distortion of pelvic structures. The most commonly encountered benign ovarian mass is a teratoma or dermoid cyst, which usually present in patients of reproductive age. These heterogenous lesions generally comprise various soft tissue, fatty, osseous, and calcific elements (Figure 17). Larger dermoids can occasionally act as a lead point in the development of adnexal torsion, which often necessitates an emergent salpingo-oophorectomy. Malignant ovarian neoplasms generally occur in the postmenopausal patient population, and rarely play a role in the development of secondary infertility. Ultrasound is generally used as a first-line imaging modality in a suspected ovarian mass, although magnetic resonance usually provides more accurate lesion characterization, and is far more accurate in local staging of disease.

\section{Disorders of the uterus}

Uterine abnormalities can be broadly subdivided into two classes, ie, disorders of the endometrium and myometrium. Endometrial disorders include synechiae or adhesions, polyps, and submucosal fibroids, all of which can be seen in the setting of secondary infertility. Myometrial disorders mainly comprise adenomyosis and leiomyomata.

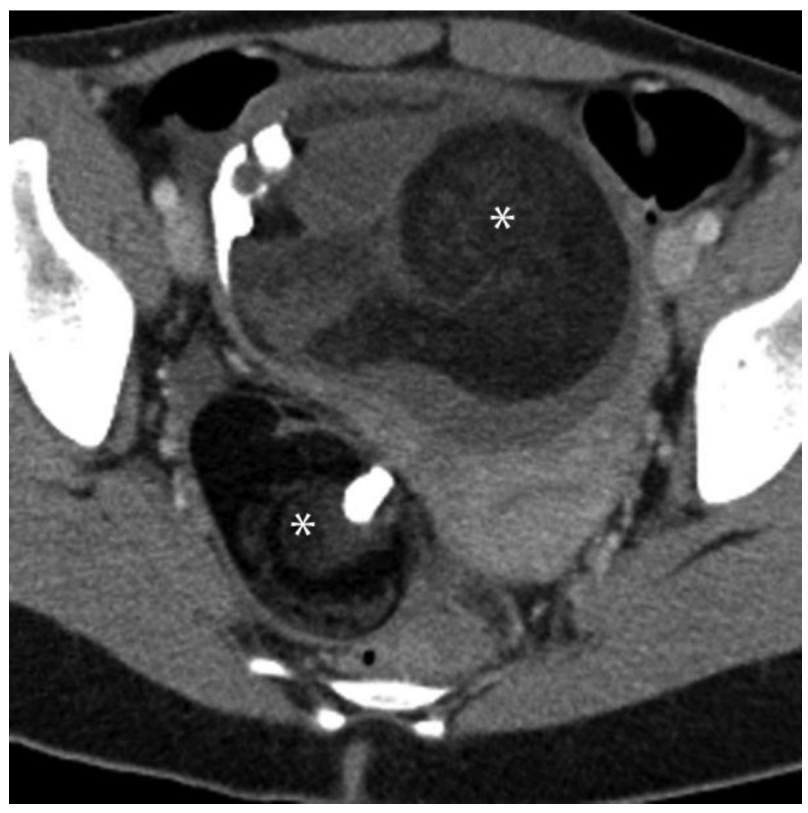

Figure 17 Bilateral ovarian teratomas. Contrast-enhanced computed tomography scan from a 23-year-old female with chronic pelvic pain and infertility demonstrates large, heterogeneous masses $\left(^{*}\right)$ arising from both adnexa. Mixed soft tissue, fatty and ossific elements are seen arising from both masses.

\section{Endometrial disorders}

Asherman syndrome refers to the presence of intrauterine adhesions that result in partial or complete obliteration of the endometrial cavity. This acquired condition is an important cause of secondary infertility, with a reported infertility rate of $43 \%$ in women studied. ${ }^{15}$ Menstrual abnormalities and recurrent pregnancy loss are likewise commonly seen. ${ }^{16}$ Asherman syndrome is generally the result of trauma to the basal layer of the endometrium from aggressive uterine curettage. Less common causes include prior C-section, myomectomy, pelvic irradiation, endometrial necrosis from uterine artery embolization, or chronic scarring from an intrauterine device. This condition is frequently diagnosed by hysterosalpingography early on in the infertility workup. Hysterosalpingography findings include multiple intracavity filling defects of variable size, with resultant distortion and limited distensibility of the endometrium. Sonohysterography has greater sensitivity than conventional hysterosalpingography for the identification of adhesions, and is often performed to confirm the presence of this condition. ${ }^{17}$ A sonohysterogram is performed by obtaining images (usually transvaginally) of the endometrium while distending the endometrial cavity with sterile saline (Figure 18). Sonohysterography findings usually demonstrate echogenic bands traversing the endometrial cavity, with limited distensibility and distortion of the uterine cavity (Figure 19). Magnetic resonance has been 


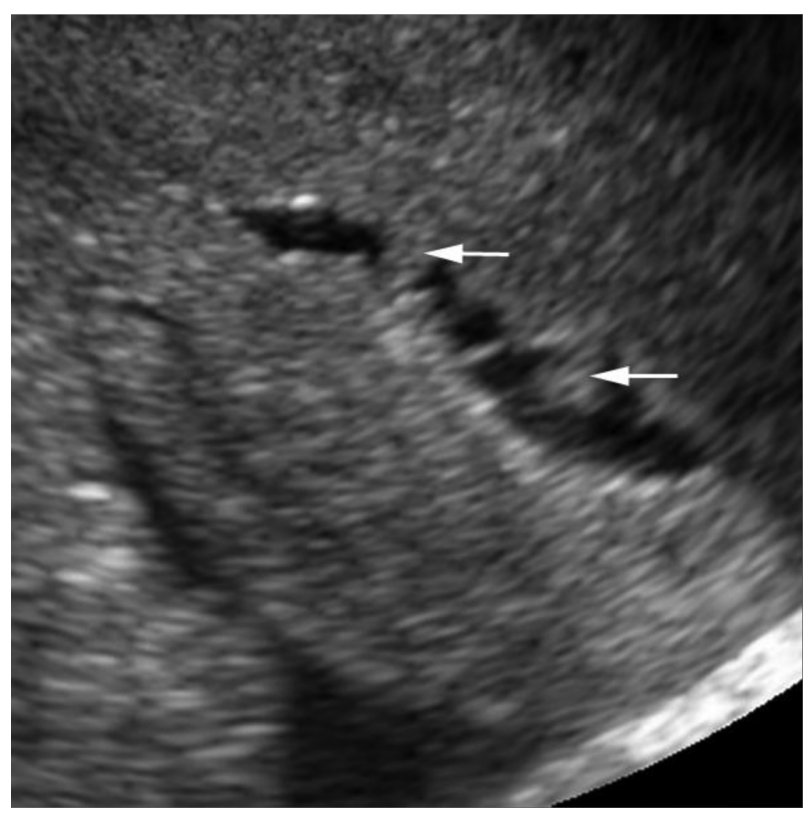

Figure 18 Asherman syndrome. Longitudinal image from a transvaginal sonohysterogram in a 33-year-old female with a history of prior dilatation and curettage. The endometrial cavity is poorly distensible and diffisely irregular in contour. Bridging echgenic foci (arrows) are noted, consistent with adhesions or synechiae.

used to assess for Asherman syndrome with variable success. The signal characteristics of intrauterine adhesions have not been examined in detail. ${ }^{16}$

Endometrial polyps are defined as polypoid foci of endometrial hyperplasia. These lesions can interfere with embryonic implantation, and are an important cause of secondary infertility.

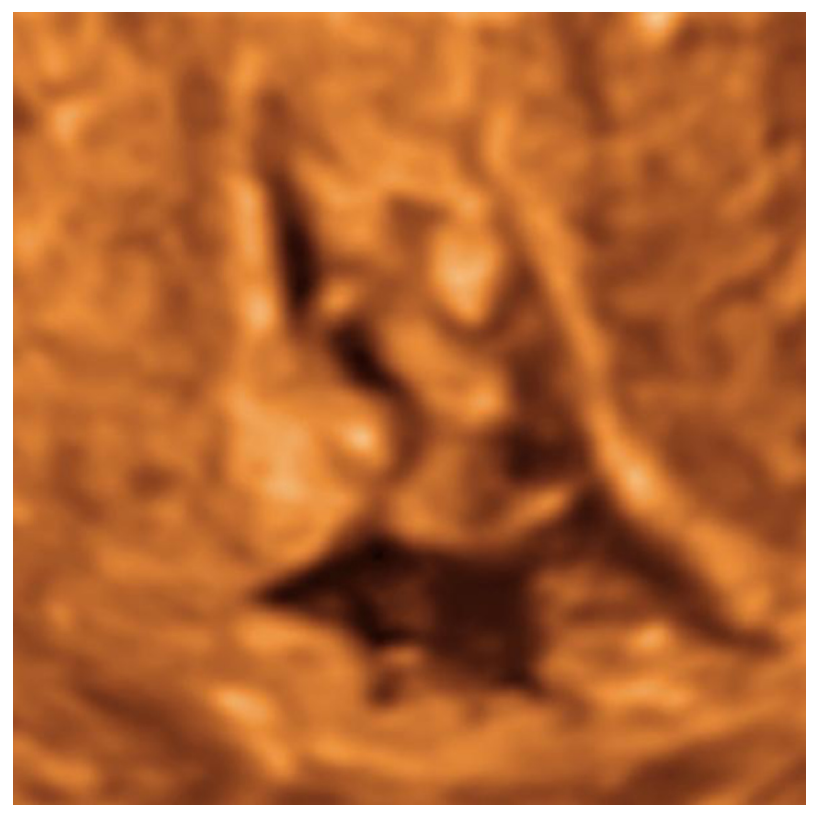

Figure 19 Asherman syndrome. Three dimensional image from a sonohysterogram in the same patient in Figure 18. Diffuse, polypoid irregularity of the endometrial cavity is noted.
Polyps can be seen in both pre- and postmenopausal women. They are more commonly seen in patients receiving tamoxifen or hormone replacement therapy. Clinical symptoms include menorrhagia and intramenstrual bleeding. These lesions tend to grow very slowly, and possess little or no malignant potential. Polyps may be seen on hysterosalpingography as discrete intraluminal filling defects. However, injected air bubbles or blood clots may mimic endometrial polyps. Additional contrast injection or patient repositioning may be required in order to establish the stationary nature of endometrial polyps. Conventional pelvic ultrasound may demonstrate focal thickening of the endometrial cavity. Endometrial polyps are readily visualized on sonohysterography as discrete, echogenic foci arising from the endometrium (Figure 20). Although multiple small polyps may mimic synechiae at sonohysterography, the lack of bridging fibrous bands and a readily distensible endometrial cavity in the former condition aid in differentiating these two entities. Color Doppler frequently demonstrates vascular flow into the polyp. Endometrial polyps are generally well visualized on magnetic resonance as focal pedunculated lesions extending into the endometrium. These lesions are usually hypointense to the adjacent endometrium on $\mathrm{T}_{2}$-weighted images (Figure 21).

\section{Myometrial disorders}

Disorders of the uterine myometrium leading to secondary infertility include adenomyosis and leiomyomata.

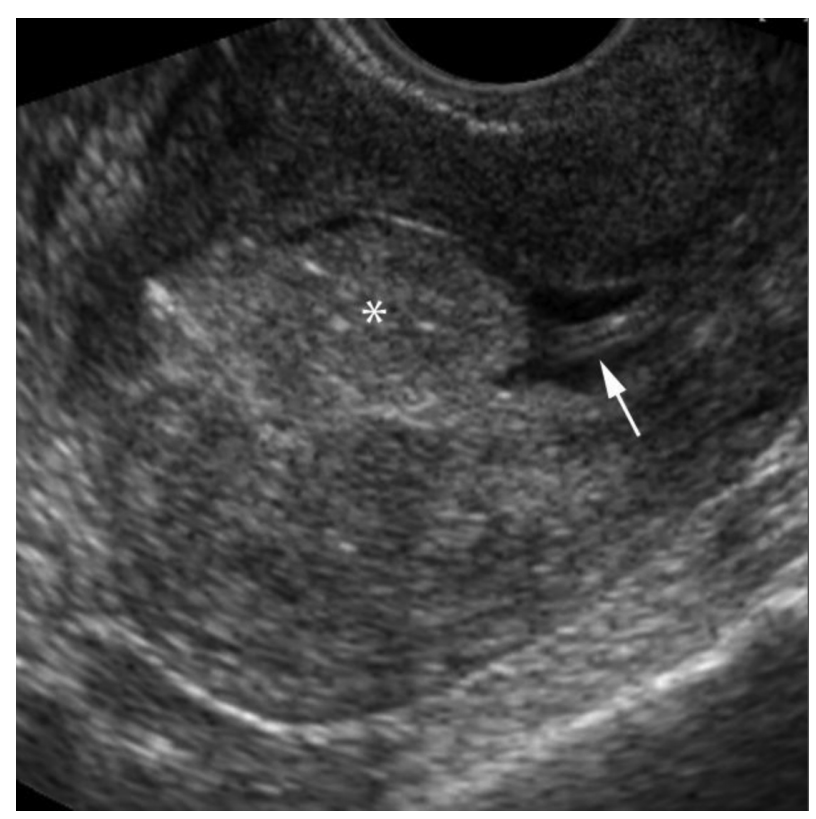

Figure 20 Endometrial polyp. Longitudinal image from a transvaginal sonohysterogram in a 35-year-old female with chronic menorrhagia and infertility. A small amount of endometrial fluid outlines a polypoid, echogenic lesion $(*)$ within the endometrium. A short segment of an intrauterine catheter is seen (arrow) within the lower uterine segment. 


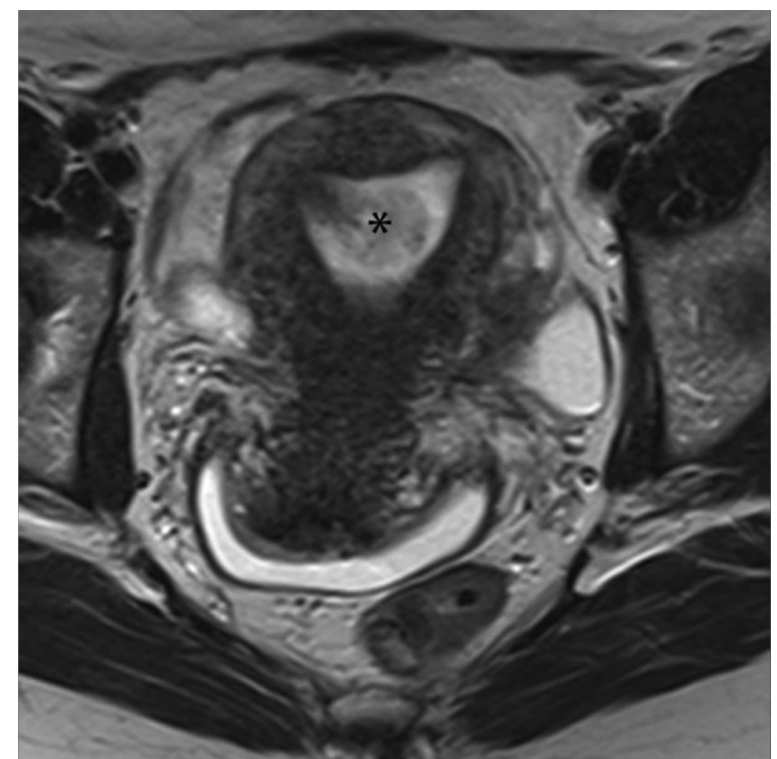

Figure 21 Endometrial polyp. Axial, $\mathrm{T}_{2}$-weighted magnetic resonance image from the same patient in Figure 20 demonstrates an intermediate signal pedunculated lesion within the endometrial cavity $(*)$.

Adenomyosis is a benign condition of the uterus that is characterized by migration of endometrial glands into the uterine myometrium. Clinical symptoms include menstrual irregularities, pelvic pain, and infertility. Adenomyosis may be a diffuse process that involves the entire myometrium, or may manifest as a focal abnormality (adenomyoma) within the myometrium. Adenomyosis is frequently associated with endometriosis, particularly in younger women..$^{18}$ It has been suggested that adenomyosis contributes to infertility due to impaired sperm transport from diminished uterine contractility.

Adenomyosis may be diagnosed by a variety of imaging modalities. Hysterosalpingography plays little role in the diagnosis of adenomyosis. The presence of irregular endometrial contour or diverticular outpouching of contrast into the myometrium may occasionally be seen at hysterosalpingography. A focal, poorly marginated collection of contrast in the myometrium may be seen in the setting of an adenomyoma (Figure 22). Ultrasound is likewise fairly nonspecific. The presence of a diffusely enlarged, or globular uterus, myometrial cysts, or an indistinct endometrial/myometrial interface may be seen. ${ }^{19}$ Magnetic resonance remains the gold standard for the diagnosis of adenomyosis. Magnetic resonance findings include diffuse or focal thickening of the hypointense junctional zone greater than $12 \mathrm{~mm}$ on $\mathrm{T}_{2}$-weighted images. Myometrial cysts are likewise commonly seen in the setting of adenomyosis, and are readily seen as small $\mathrm{T}_{2}$ hyperintense foci (Figure 23).

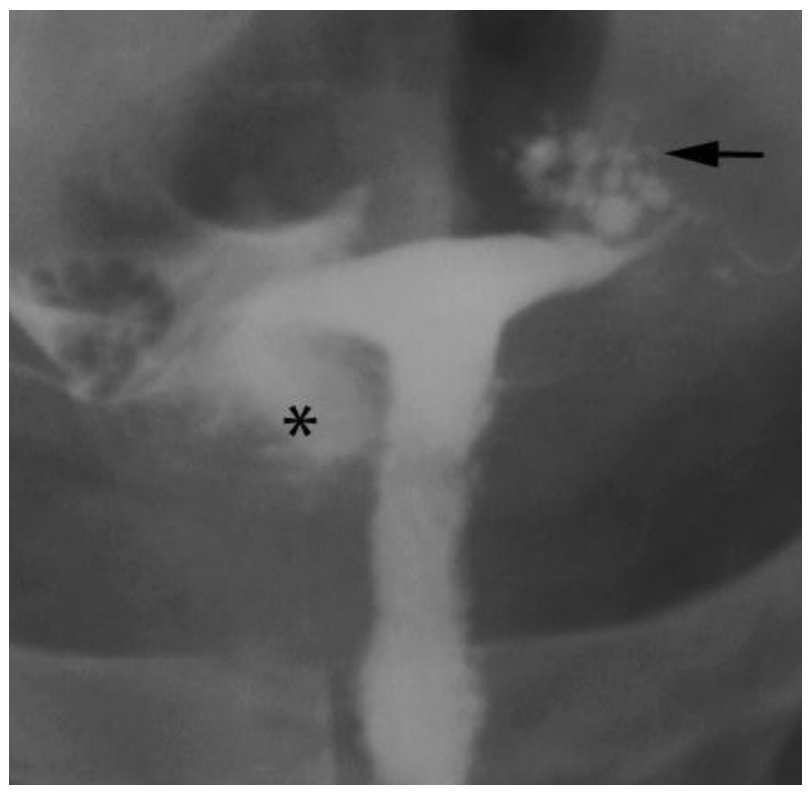

Figure 22 Adenomyosis. Hysterosalpingography from a 27-year-old female with chronic pelvic pain and infertility. An ill-defined contrast collection is noted $(*)$ surrounding the right uterine cornu. Additionally, multiple diverticular outpouchings are noted arising from the left cornu (arrow).

Uterine leiomyomas are the most common benign masses of the uterus. These lesions are estrogen-sensitive, and tend to enlarge over time. They are a well established cause of secondary infertility. Leiomyomas may occur in any portion of the uterus, and are classically subdivided as submucosal, intramural, and subserosal in location. Submucosal leiomyomas

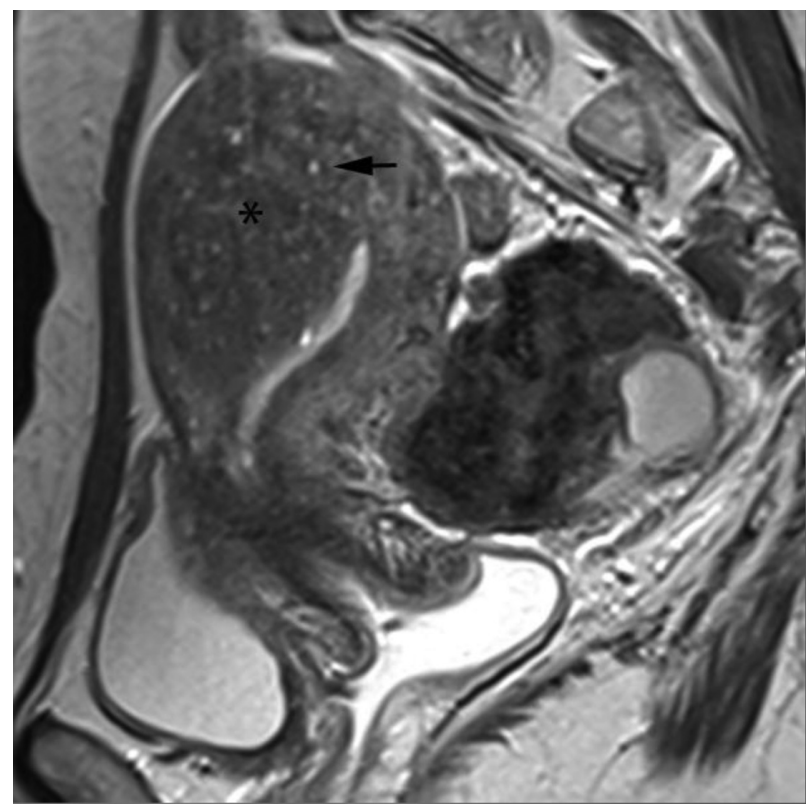

Figure 23 Adenomyoma. Sagittal, $T_{2}$-weighted magnetic resonance image from a 25 -year-old female with dysmenorrhea and infertility. A poorly marginated hypointense mass $(*)$ is seen within the anterior myometrium. Punctate hyperintense foci are likewise noted (arrow), consistent with focal endometrial rests. 
have the strongest association with impaired fertility due to an inherently higher degree of endometrial distortion. They may be broad-based, polypoid, or highly pedunculated (Figure 24). As with endometrial polyps, these lesions may result in infertility due to impaired embryonic implantation. ${ }^{20}$

Leiomyomas are readily diagnosed by a variety of imaging modalities. Submucosal and large intramural leiomyomas commonly distort the endometrial cavity at hysterosalpingography, whereas subserosal lesions may not be detected. Leiomyomas may have a variable appearance at ultrasound. Although they are generally hypoechoic relative to the adjacent myometrium, they may occasionally be iso- or even hyperechoic. Magnetic resonance remains the most accurate imaging modality for the detection and characterization of uterine leiomyomas. The lesions are generally hypointense on both $\mathrm{T}_{1}$ - and $\mathrm{T}_{2}$-weighted images (Figure 25).

Müllerian duct anomalies are congenital defects of the uterus. It is estimated that $3 \%$ of women with recurrent pregnancy losses have a congenital uterovaginal anomaly. ${ }^{21}$ These anomalies are an important cause of primary infertility, but are less important in the setting of secondary infertility. These anomalies are generally classified according to the American Fertility Society, and comprise seven classes: I, uterine hypoplasia and agenesis; II, unicornuate uterus; III, uterus didelphys; IV, bicornuate uterus; V, septate uterus; VI, arcuate uterus; and VII, diethylstilbestrol-related

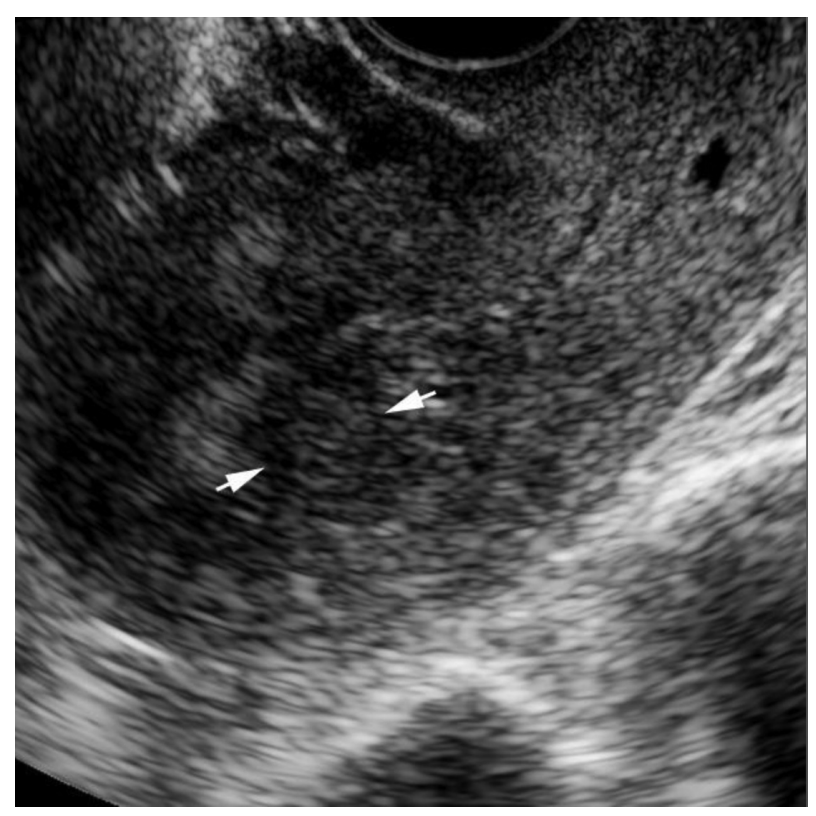

Figure 24 Submucosal leiomyoma. Longitudinal, transvaginal pelvic sonogram from a 33-year-old female with a history of dysmenorrhea and infertility. A polypoid hypoechoic lesion (arrows) is seen protruding into the endometrial cavity.

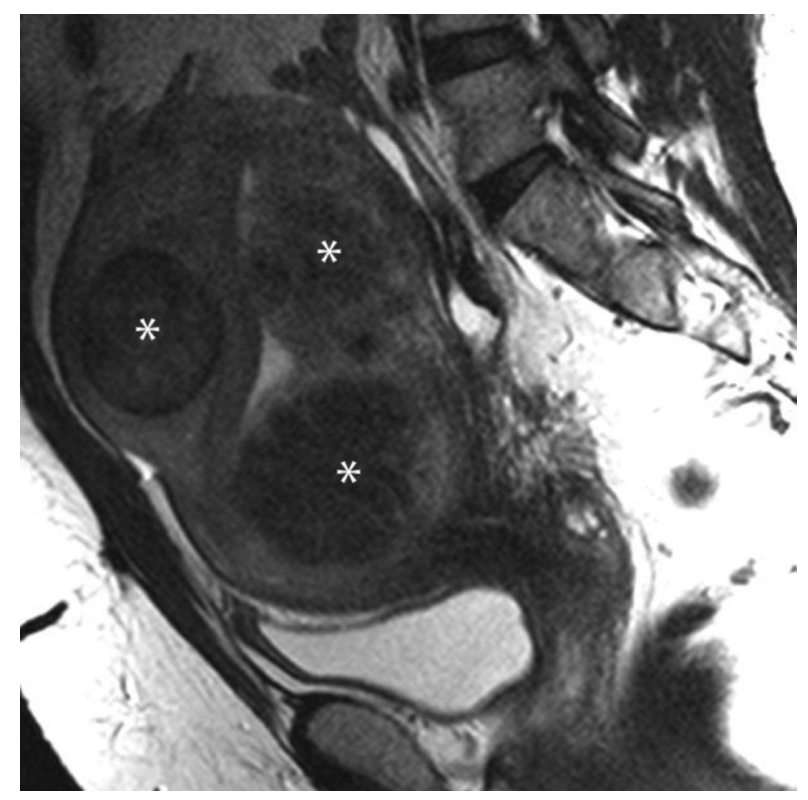

Figure 25 Uterine leiomyomata. Sagittal $\mathrm{T}_{2}$-weighted magnetic resonance image from a 38-year-old woman with chronic pelvic pain. Sharply marginated, hypointense masses $(*)$ are noted arising within the myometrium with resultant distortion of the posterior aspect of the endometrium.

anomalies. Accurate characterization of these anomalies is crucial, because treatment options and reproductive outcomes vary depending on the type and severity of the anomaly. Hysterosalpingography and ultrasound may both suggest the presence of Müllerian duct anomalies

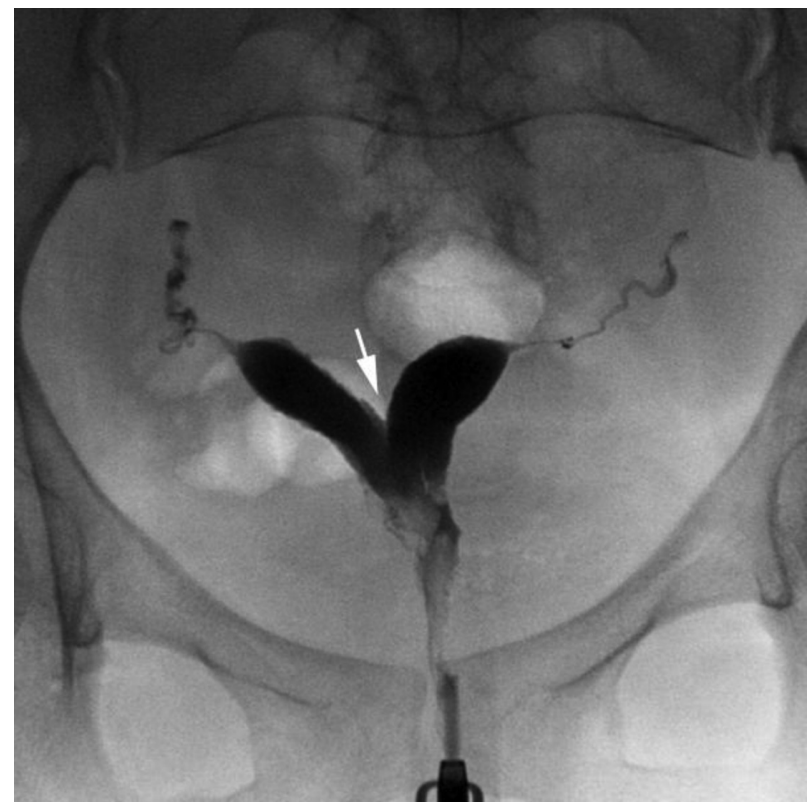

Figure 26 Septate uterus. Hysterosalpingography from a 38-year-old female with a history of recurrent miscarriages. A deep cleft is noted (arrow) involving the fundic portion of the endometrium. While this image is suggestive of a Müllerian duct anomaly, a more precise diagnosis is not feasible without visualization of the external uterine contour. 
(Figure 26). However, magnetic resonance provides the most accurate means of anatomic characterization, and is considered the gold standard amongst available imaging modalities (Figure 27).

\section{Disorders of the cervix}

Cervical abnormalities that contribute to female infertility include cervical factor infertility and cervical stenosis. Cervical factor infertility entails an inadequate amount of cervical mucus, which is thought to account for up to $10 \%$ of female infertility. ${ }^{21}$ This condition is rarely seen in the setting of secondary infertility. Additionally, the diagnosis is made clinically, and does not involve imaging.

Cervical stenosis may be a congenital or acquired condition. Congenital cervical stenosis is rarely, if ever, seen in the setting of secondary infertility. The acquired form can lead to secondary infertility, and is generally the result of infection or trauma. Risk factors include prior cervical instrumentation, such as previous cone biopsy, cryotherapy, or laser treatment. Cervical stenosis can result in obstruction of menstrual flow with resulting amenorrhea, dysmenorrhea, and potential infertility due to the blockage of sperm in the endometrial canal. Clinical symptoms include cyclic pelvic pain from hematometra and hematosalpinx. Urinary retention and constipation

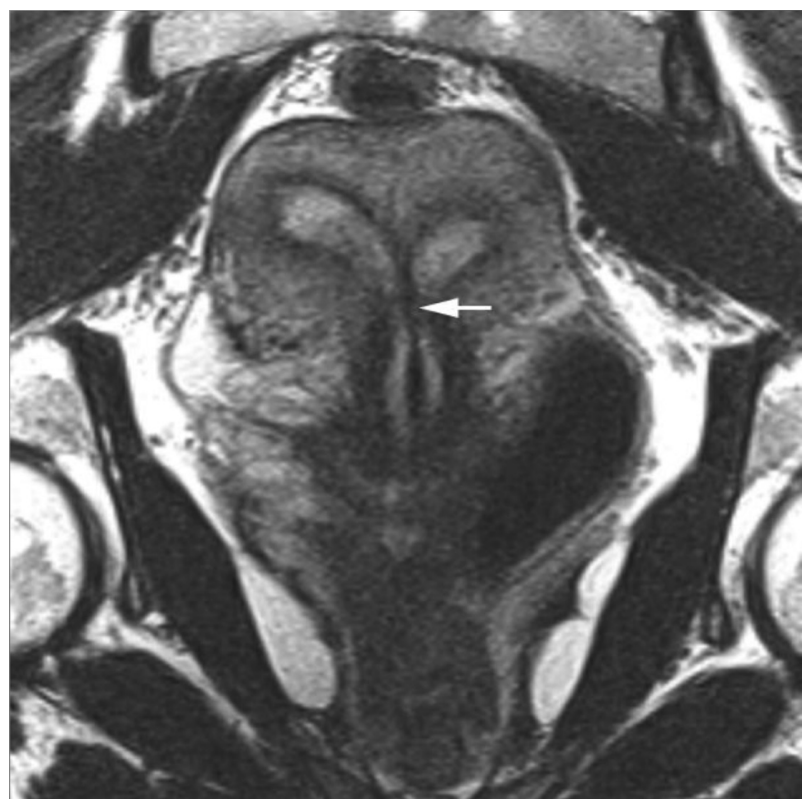

Figure 27 Septate uterus. Oblique axial $T_{2}$-weighted magnetic resonance image through the long axis of the uterine in the same patient in Figure 26. A hypointense midline septum is noted dividing the endometrial cavity (arrow). The flat external fundal contour of the uterus is in keeping with the diagnosis of a septate uterus. may occasionally be seen from extrinsic compression due to severe hematometra. Women of reproductive age are at increased risk of developing endometriosis due to retrograde menstruation from cervical obstruction. The incidence of uterine infections is likewise increased. The diagnosis of cervical stenosis can be established at hysterosalpingography by diffuse narrowing or obstruction of the endocervical canal if contrast material is introduced at the level of the cervical os. Ultrasound or magnetic resonance may indirectly infer the presence of cervical stenosis by the presence of a dilated, fluid-filled endometrial cavity (Figures 28 and 29). Treatment options include dilation and evacuation of the dilated endometrial canal for shortterm relief. Catheter placement for long-term drainage or hysteroscopic excision of cervical tissue may likewise be performed.

\section{Disorders of the peritoneum}

Endometriosis is a relatively common etiology of chronic pelvic pain, and accounts for a significant percentage of both primary and secondary female infertility. The condition is characterized by the extrauterine presence of endometrial glands and stroma that generally occurs as a result of retrograde menstruation. An estimated $30 \%-50 \%$ of women with endometriosis are infertile, and $20 \%$ of infertile women have endometriosis. ${ }^{22}$ Endometriosis affects women almost

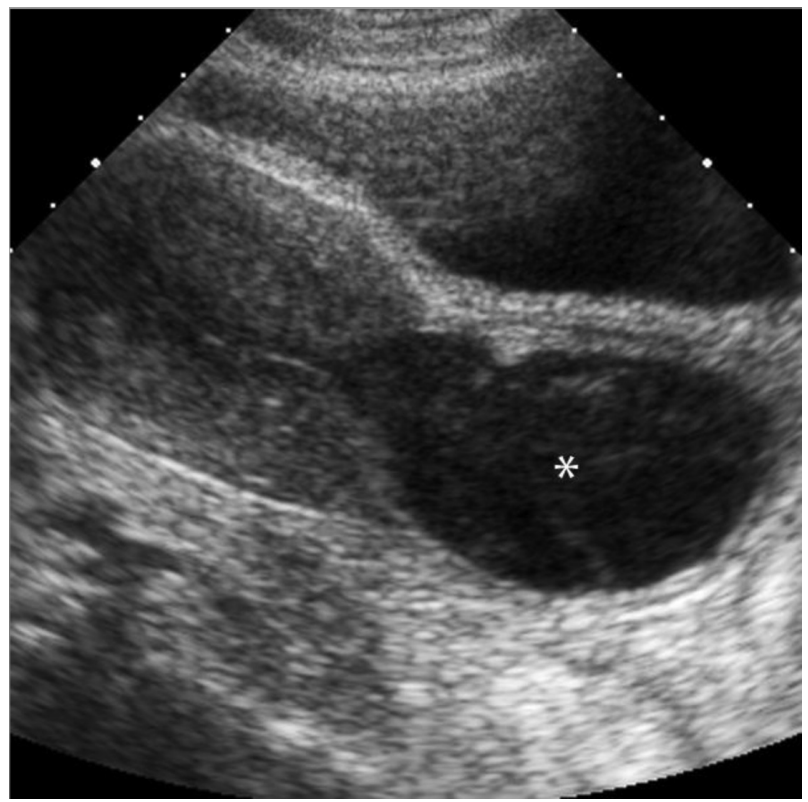

Figure 28 Hematocolpos. Longitudinal image from a transabdominal pelvic sonogram in a 34-year-old female with oligomenorrhea and infertility. A large, mildly heterogeneous fluid collection is noted (*) expanding the endocervical canal in this patient with radiation-induced cervical stenosis. 


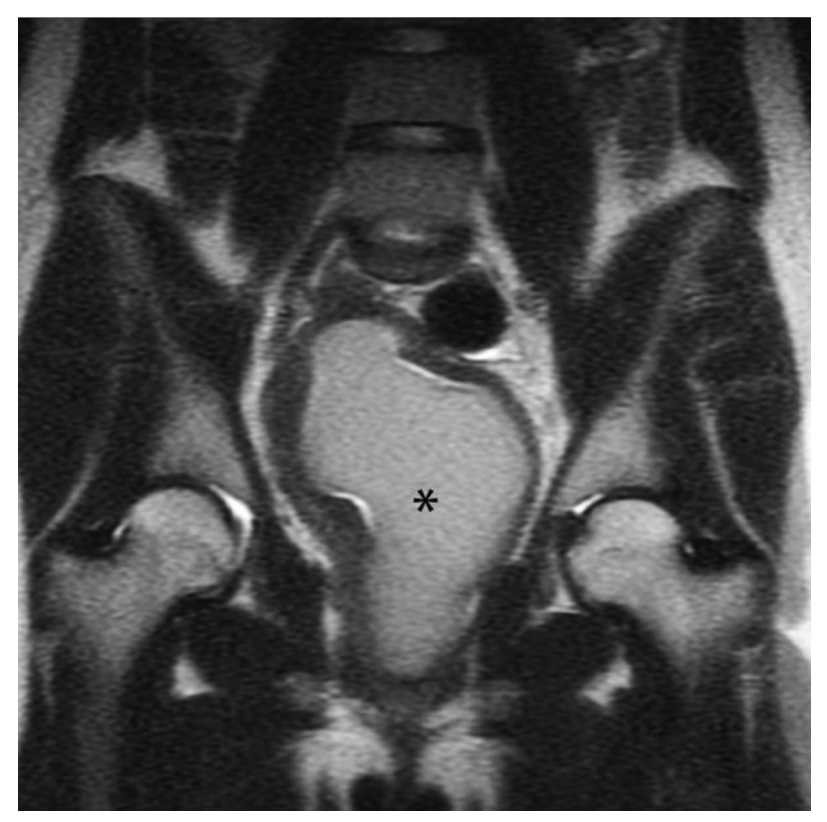

Figure 29 Hematometrocolpos. Coronal $\mathrm{T}_{2}$-weighted magnetic resonance image in a $4 \mathrm{I}$-year-old female with a history of prior cervical cryotherapy. A large intrauterine fluid collection is noted $(*)$ with marked expansion of the endometrial cavity.

exclusively during their reproductive years. Symptoms include chronic, often cyclic, pelvic pain, dysmenorrhea, dyspareunia, and irregular bleeding, although a large percentage of women are asymptomatic. The ovaries are the most common site of implantation. Multiple peritoneal sites include uterine ligaments, cul de sac, pelvic peritoneum reflected over the uterus, fallopian tubes, rectosigmoid colon, and bladder. Rare extraperitoneal sites include lungs and the central nervous system.

The disease is manifested in the form of focal implants or endometriomas, or small endometrial deposits or implants. Endometriomas are thick-walled, complex hemorrhagic cysts with debris or "chocolate cysts". These lesions may be solitary or multiple. Endometrial implants are generally small, punctate hemorrhagic foci on the peritoneal surface. Ultrasound and magnetic resonance are the primary imaging modalities commonly employed for the detection and characterization of endometriosis. Hysterosalpingography may reveal peritubal adhesions, which are manifested as abnormal accumulations of contrast material adjacent to the distal or ampullary ends of the fallopian tubes. Peritubal adhesions are likewise seen in the setting of chronic pelvic inflammatory disease, as previously discussed. Although CT may reveal nonspecific, soft tissue attenuation masses in the setting of endometriosis, the modality plays little role in the evaluation of this condition.
The sonographic appearance of an endometrioma is variable. The most common appearance is a focal mass containing diffuse, homogeneous, low-level internal echoes with hyperechoic mural foci (Figure 30 ). ${ }^{23}$ Punctate mural calcifications may occasionally be seen. Focal areas of clot may mimic a solid mass, although color Doppler interrogation demonstrates no flow within these lesions. Peritoneal plaques or focal implants are not well visualized by ultrasound. Peritoneal plaques may occasionally be seen as hypoechoic or anechoic foci that can mimic fluid in the posterior cul de sac. ${ }^{24}$ There may be pelvic adhesions with resultant close proximity of the ovaries to the uterus, otherwise known as "kissing ovaries".

Magnetic resonance remains the imaging modality of choice for the detection and characterization of endometriosis. Endometriomas are characteristically hyperintense on $\mathrm{T}_{1}$-weighted images due to the presence of internal blood products. The lesions remain hyperintense on fat-saturated images (Figure 31). $\mathrm{T}_{1}$-weighted images may likewise demonstrate a hematosalpinx, which appears as a tubular, hyperintense structure adjacent to the ovary (Figure 32). Endometriomas demonstrate heterogeneous signal intensity on $\mathrm{T}_{2}$-weighted images, a feature commonly referred to as $\mathrm{T}_{2}$ "shading" (Figure 33). There is little to no enhancement of endometriomas following contrast administration. Delayed enhancement can rarely be seen. Endometrial plaques are frequently seen as

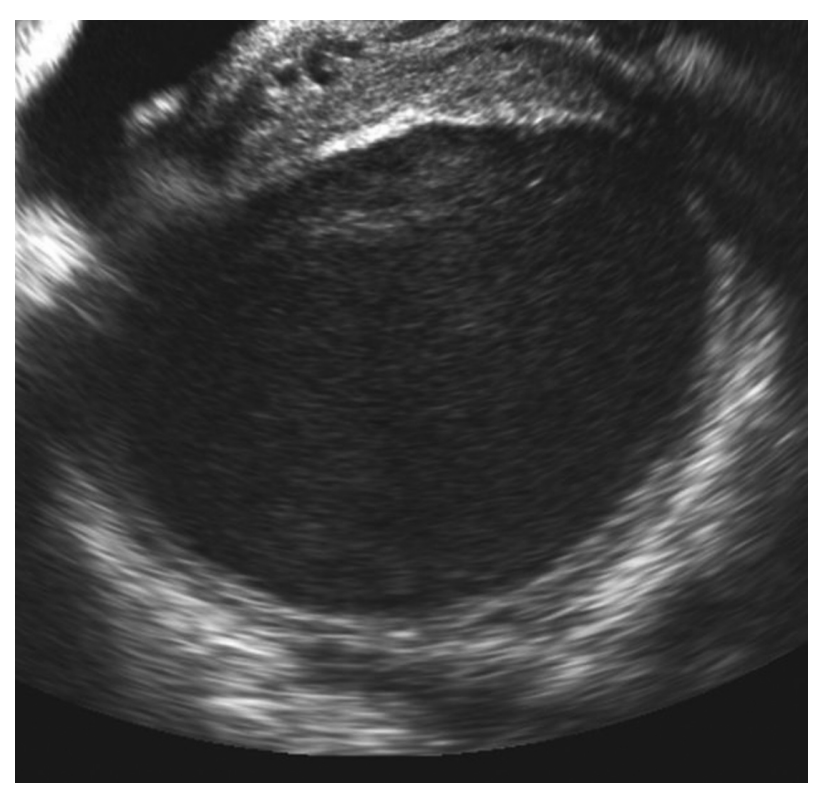

Figure 30 Endometrioma. Transverse image from a transvaginal pelvic sonogram in a 36-year-old female with chronic pelvic pain and infertility. A well circumscribed mass in noted arising from the right adnexa which contains homogeneous, low level internal echoes. 


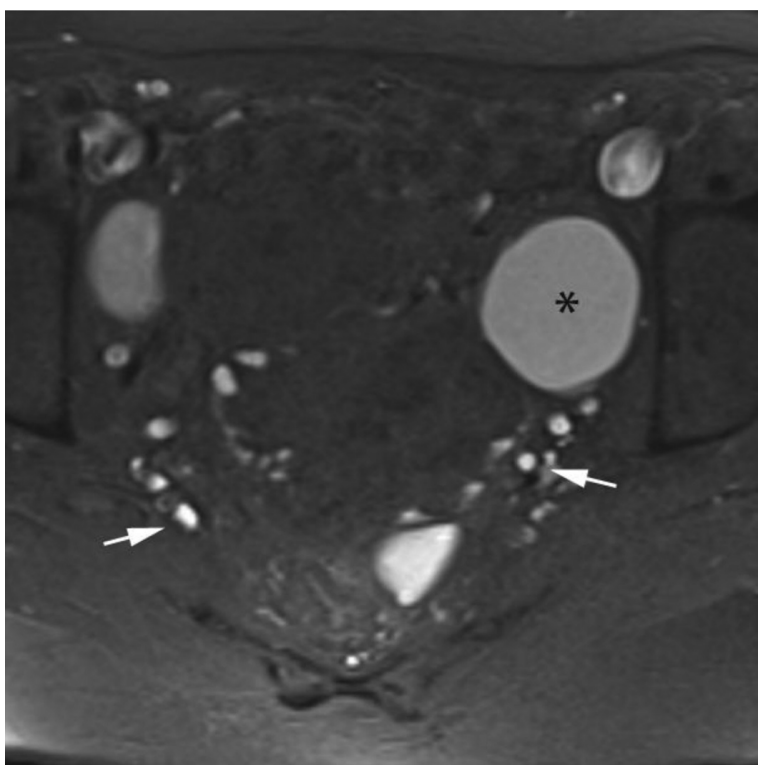

Figure 3 I Endometriosis. Axial $T_{1}$-weighted magnetic resonance image with fat saturation in the same patient from Figure 30. A uniformly hyperintense lesion is noted along the left pelvic sidewall $(*)$, consistent with a prominent endometrioma. Several other subcentimeter hyperintense foci are noted throughout the pelvis (arrows), consistent with small endometrial implants.

focal, hyperintense foci on $\mathrm{T}_{1}$-weighted images. These small implants are often isointense to fat on conventional $\mathrm{T}_{1}$-weighted images. Therefore, it is imperative that fat saturation techniques be employed in order to identify endometrial implants reliably.

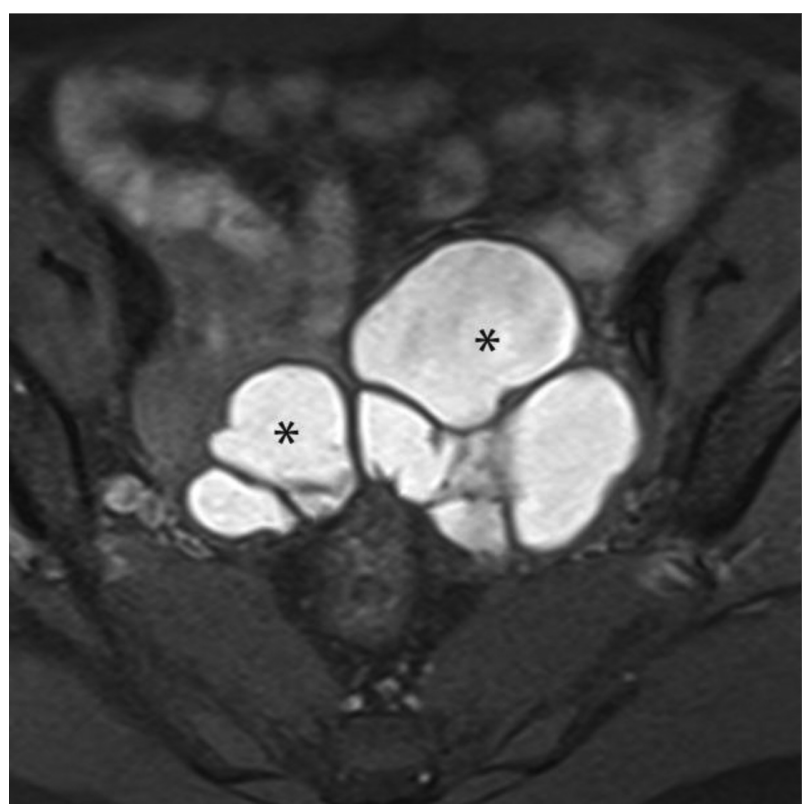

Figure 32 Endometriosis with bilateral hematosalpinges. Axial $T_{1}$-weighted magnetic resonance image with fat saturation in a 34-year-old female with a known history of chronic endometriosis. Bilateral hyperintense tubular structures are noted $(*)$, consistent with hematosalpinges.

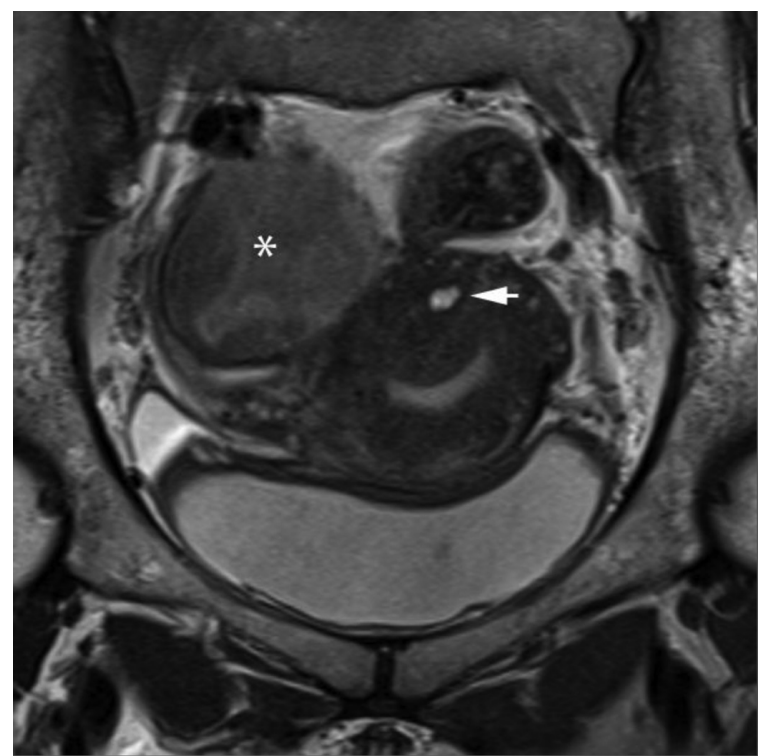

Figure 33 Endometriosis. Coronal $\mathrm{T}_{2}$-weighted magnetic resonance image of the pelvis in a 38-year-old female with chronic pelvic pain and infertility. A heterogeneous, intermediate signal mass is noted arising from the right adnexa $(*)$, consistent with endometrioma. Additionally, there is heterogeneous thickening of the uterine junctional zone with a small hyperintense focus in the myometrium (arrow). These findings are consistent with adenomyosis.

\section{Conclusion}

There are multiple causes of secondary female infertility which include a variety of tubal, ovarian, uterine, cervical, and peritoneal disorders. Hysterosalpingography generally serves as a baseline imaging modality for the evaluation of secondary infertility. However, other complementary imaging modalities have been developed in recent years that have become indispensible in the assessment of secondary female infertility. Radiologists must be familiar with the strengths and weaknesses of these techniques in order to ensure that patients with secondary infertility are appropriately managed.

\section{Disclosure}

The authors report no conflicts of interest in this research.

\section{References}

1. Chandra A, Martinez GM, Mosher WD, et al. Fertility, family planning, and reproductive health of U S. women: Data from the 2002 National Survey of Family Growth. Vital Health Stat 23. 2005;125: $1-160$.

2. Wright VC, Chang J, Jeng G, et al. Assisted reproductive technology surveillance - United States, 2004. MMWR Surveill Summ. 2007; $56: 1-22$.

3. Imaoka I, Wada A, Matsuo M, et al. MR imaging of disorders associated with female infertility: Use in diagnosis, treatment, and management. Radiographics. 2003;23:1401-1421.

4. Reis MM, Soares SR, Cancado ML, et al. Hysterosalpingo contrast sonography (HyCoSy) with SH U 454 (Echovist) for the assessment of tubal patency. Hum Reprod. 1998;13:3049-3052. 
5. Carrascosa PM, Capunay C, Vallejos J, et al. Virtual hysterosalpingography: A new multidetector CT technique for evaluating the female reproductive system. Radiographics. 2010;30:643-663.

6. Rouanet de Lavit JP, Maubon AJ, Thurmond AS. MR hysterography performed with saline injection and fluid attenuated inversion recovery sequences: Initial experience. Am J Roentgenol. 2000;175:774-776.

7. Simpson WL, Beitia LG, Mester J. Hysterosalpingography: A reemerging study. Radiographics. 2006;26:419-431.

8. Winfield A, Pittaway D, Maxson W, et al. Apparent cornual occlusion in hysterosalpingography: Reversal by glucagon. Am J Roentgenol. 1982;139:525-527.

9. McCormack WM. Pelvic inflammatory disease. N Engl J Med. 1994; 330:115-119.

10. Soper DE. Pelvic inflammatory disease. Infect Dis Clin North Am. 1994; 8:821-840.

11. Sam JW, Jacobs JE, Birnbaum BA. Spectrum of CT findings in acute pyogenic pelvic inflammatory disease. Radiographics. 2002; 22:1327-1234.

12. Krysiewicz S. Infertility in women: Diagnostic evaluation with hysterosalpingography and other imaging techniques. Am J Roentgenol. 1992;159:253-261.

13. Romo LV, Clarke PD. Fitz-Hugh-Curtis syndrome: Pelvic inflammatory disease with an unusual CT presentation. J Comput Assist Tomogr. 1992; 16:832-833.

14. Ricardo A, Enrico C, Didier D, et al. The Androgen Excess and PCOS Society criteria for the polycystic ovary syndrome: The complete task force report. Fertil Steril. 2009;91:456-488.
15. Schenker JG, Margalioth EJ. Intrauterine adhesions: An updated appraisal. Fertil Steril. 1982;37:593-610.

16. Yu D, Wong YM, Cheong Y, et al. Asherman syndrome - one century later. Fertil Steril. 2008;89:759-779.

17. O’Neill MJ. Sonohysterography. Radiol Clin North Am. 2003;41: 781-797.

18. Kunz G, Beil D, Huppert P, et al. Adenomyosis in endometriosis prevalence and impact on fertility. Evidence from magnetic resonance imaging. Hum Reprod. 2005;20:2309-2316.

19. Reinhold C, Tafazoli F, Mehio A, et al. Uterine adenomyosis: Endovaginal US and MR imaging features with histopathologic correlation. Radiographics. 1999;19:S147-S160.

20. Thurmond AS. Imaging of female infertility. Radiol Clin North Am. 2003;41:757-767.

21. Steinkeler JA, Woodfield CA, Lazarus E, et al. Female infertility: A systematic approach to radiologic imaging and diagnosis. Radiographics. 2009;29:1353-1370.

22. Eskenazi B, Warner ML. Epidemiology of endometriosis. Obstet Gynecol Clin North Am. 1997;24:235-258.

23. Woodward PJ, Sohaey R, Mezzetti TP. Endometriosis: Radiologicpathologic correlation. Radiographics. 2001;21:193-216.

24. Friedman H, Vogelzang RL, Mendelson EB, et al. Endometriosis detection by US with laparoscopic correlation. Radiology. 1985;157: 217-220.
Reports in Medical Imaging

\section{Publish your work in this journal}

Reports in Medical Imaging is an international, peer-reviewed, open access journal publishing original research, reports, reviews and commentaries on all areas of medical imaging. The manuscript management system is completely online and includes a very quick and fair peer-review system, which is all easy to use.

\section{Dovepress}

Visit http://www.dovepress.com/testimonials.php to read real quotes from published authors. 